\title{
Influence of thermo-gravitational convection to temperature fields in small surroundings of the heat source
}

\author{
Alexander Kondakov ${ }^{1, *}$ \\ ${ }^{1}$ National Research Tomsk Polytechnic University, 634050 Tomsk, Russia
}

\begin{abstract}
The mathematical modeling of temperature and velocity fields in the system "the heat source - environment - the object of heating" was conducted. The impact assessment of thermo-gravitational convection to the temperature field in comparison with the model of conductive heat transfer was done.
\end{abstract}

\section{Introduction}

When developing technologies of formation of thermal conditions of rooms, as a rule, methods which are based on relatively simple balancing equations, are used. When outputting the last ones it was supposed that temperature in the room and conditions of heat transfer between the object and the heat source do not change in space and time.

In the real practice temperature differentials can obtain a few degrees in the concerned conditions, and heat removal capacity from the heating object is higher in two-three times even in the conditions of natural convection than in the conditions of conduction. This depends on geometrical and operating characteristics of the system "the heat source environment - the object of heating".

Because of these reasons, enhancement of advantageous thermal operation ensuring systems can succeed when describing spatial temperature fields and the air velocity during the operation of thermo-gravitational convection in the concerned system. These methods have not being developed till now. In this regard the purpose of the work is numerical study of heat transfer in the system "the heat source - environment - the object of heating" and getting of temperature and velocity fields for evaluation of heat transfer characteristics change in the natural convection.

\section{Formulation of the problem}

The problem of heat transfer in the conditions of natural convection between heat source and the heating object is considering.

\footnotetext{
* Corresponding author: kondakov-alexandr@,rambler.ru
} 
Two parallel vertical plates with constant temperatures $T_{l}$ and $T_{0}$, the height $H$ and which are disposed in the distance $L$ one to another are accepted as the source and the object (Fig. 1).

There is gas with constant viscosity, heat conduction and specific heat capacity between the source and the object. When formulating the problem, assumption about constancy of liquid density except lift force estimation was accepted. Here linear dependence of density from temperature is used.

The movement of the gas between the source and the heating object is considered as laminar. This takes a place at relatively small differences of temperatures and moderate object's sizes.

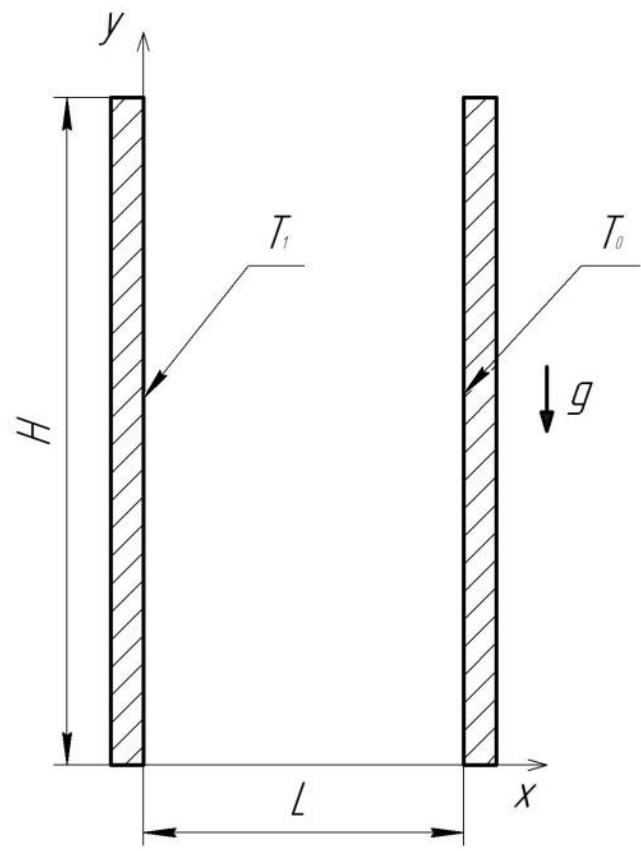

Fig. 1. Formulation of the problem.

The movement of the gas between the source and the heating object is considered as laminar. This takes a place at relatively small differences of temperatures and moderate object's sizes.

\section{Mathematical model and study technique}

The problem was solved by analogy with [3]. Two-dimensional unsteady system of differential Navier-Stokes equations in variables eddy $(w)$ - streamline function $(\psi)$ in Boussinesq approximation was used.

The following equations was accepted to lead the system to nondimensional form:

$$
\begin{gathered}
x=\frac{X}{L} \\
y=\frac{Y}{L}
\end{gathered}
$$




$$
\begin{gathered}
u=\frac{U}{V_{0}} \\
v=\frac{V}{V_{0}} \\
\theta=\frac{T-T_{0}}{\Delta T} \psi=\frac{\Psi}{V_{0} L} \\
w=\frac{\Omega}{V_{0} / L} \\
V_{0}=\sqrt{g \beta \Delta T L} \\
\Delta T=T_{1}-T_{0}
\end{gathered}
$$

Here: $X, Y$ - coordinates, $m ; x, y$ - nondimensional coordinates; $L$ - width of the calculation area $m ; U, V$ - horizontal and vertical components of the velocity vector, $\mathrm{m} / \mathrm{s}$; $u, v$ - nondimensional components of the velocity; $V_{0}$ convection velocity, $\mathrm{m} / \mathrm{s} ; \theta$ nondimensional temperature; $T$ - temperature, $K ; g$ - acceleration of gravity, $\mathrm{m} / \mathrm{s}^{2} ; \beta-$ volume expansivity, $K^{-1} ; \Psi-$ stream function, $\mathrm{m}^{2} / \mathrm{s} ; \psi-$ nondimensional stream function; $\Omega$ - eddy, $s^{-1} ; w$ - nondimensional analogue $\Omega ; \sigma$-iteration parameter, $s$.

$$
\left\{\begin{array}{l}
\frac{\partial w}{\partial t}+u \frac{\partial w}{\partial x}+v \frac{\partial w}{\partial y}=\frac{1}{\sqrt{G r}}\left(\frac{\partial^{2} w}{\partial x^{2}}+\frac{\partial^{2} w}{\partial y^{2}}\right)+\frac{\partial \theta}{\partial x} \\
\frac{\partial \psi}{\partial \sigma}=\frac{\partial^{2} \psi}{\partial x^{2}}+\frac{\partial^{2} \psi}{\partial y^{2}}-w \\
\frac{\partial \theta}{\partial t}+u \frac{\partial \theta}{\partial x}+v \frac{\partial \theta}{\partial y}=\frac{1}{\operatorname{Pr} \sqrt{G r}}\left(\frac{\partial^{2} \theta}{\partial x^{2}}+\frac{\partial^{2} \theta}{\partial y^{2}}\right)
\end{array}\right.
$$

Initial and boundary conditions for the system:

$$
\begin{gathered}
t=0: w(x, y, 0)=\psi(x, y, 0)=\theta(x, y, 0) \\
x=L, 0<y<H: \psi(L, y, t)=\theta(L, y, t)=0,\left.\frac{\partial \psi}{\partial x}\right|_{x=L}=0 ; \\
x=0,0<y<H: \psi(0, y, t)=\theta(0, y, t)=0,\left.\frac{\partial \psi}{\partial x}\right|_{x=0}=0 ; \\
y=H, 0<x<L:\left.\frac{\partial^{2} \psi}{\partial y^{2}}\right|_{y=H}=\left.\frac{\partial^{2} \theta}{\partial y^{2}}\right|_{y=H}=0 ;
\end{gathered}
$$




$$
y=0,0<x<L:\left.\frac{\partial^{2} \psi}{\partial y^{2}}\right|_{y=0}=\left.\frac{\partial^{2} \theta}{\partial y^{2}}\right|_{y=0}=0 .
$$

The discretization of differential equations was conducted by finite difference method on the constant mesh with the number of nodes $100 \times 200$ on the base of second-order Taylor's approximation of the required functions (the choice of computation mesh dimension is determined by approximation scheme [3]).

The system of algebraic equations was calculated by the method of alternating directions [4] on each step by time.

Permanence of temperature and velocity fields on two adjacent time layers was taken as the convergence criteria (with the divergence no more than $10^{-8}$ for all variables).

\section{Results of numerical modelling}

Calculations was conducted in the computational domain size $H \times L=2 \times 1$. Air is accepted as working fluid between the heating object and the source $(\operatorname{Pr}=0.71)$.

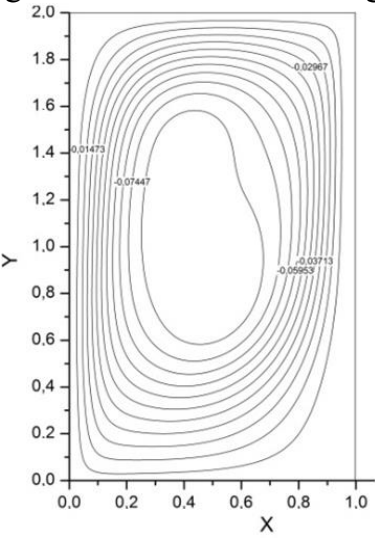

(a)

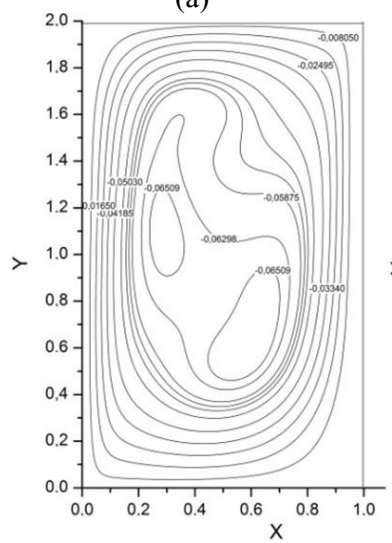

(c)

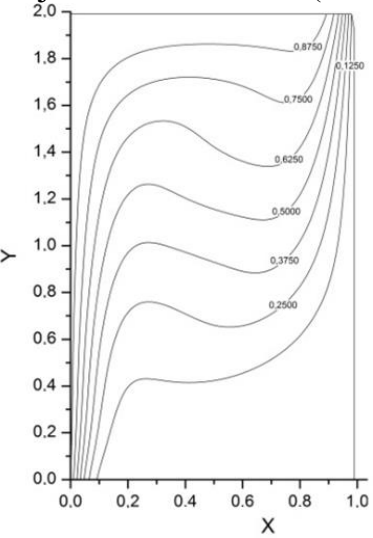

(b)

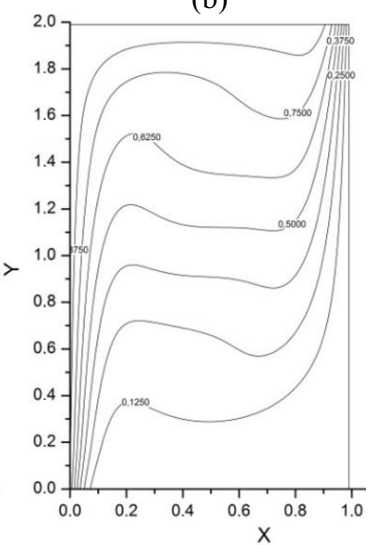

(d) 


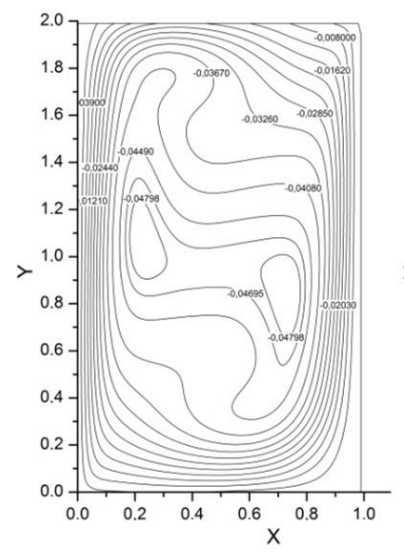

(e)

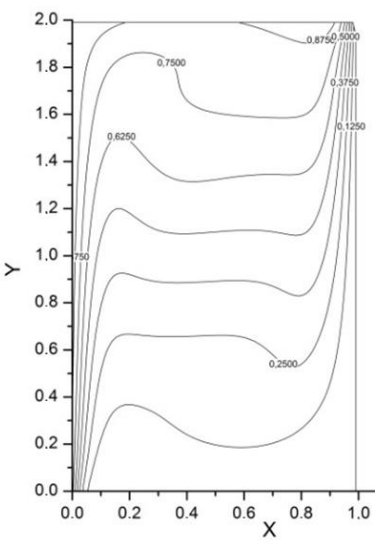

(f)

Fig. 2. Streamline functions (a.c. e.) and temperature (b. d.f.) at different Grashof numbers: $G r=10^{5}(a . b) ; G r=2 \cdot 10^{5}(c . d) ; G r=5 \cdot 10^{5}(e . f)$.

Results of numerical modelling of air movement between the object and the source at numbers $G r=10^{5} ; 2 \cdot 10^{5} ; 5 \cdot 10^{5}$ (steady flow) are shown on the figure 2 . They are presented as isotherms (a.c.e) and streamline functions (b. d. f).

When gas is near with the source, it heats. As the result, temperature gradient in the crosscut direction of the calculation area is appeared. Nonuniformly heated liquid starts to move under the influence of gravity; lift movement takes place along the left bound and the lowering movement - along the right bound (the heating object).

It was established that circulating gas motion in the considering area occurs at Grashof number $G r=10^{5}$.

Growth of Grashof (difference raising of the object's and the source's temperatures) number leads to intensification of heat transfer; secondary streams start to forming inside the main eddy (c. e). These processes also influence on the behavior of isotherms when the gas moves in the considering system. The increase of temperature gradients' value on the whole calculation area is shown in the figure $2(d . f)$.

Temperature distributions between the object and the source in the midsection $(y=1)$ are presented on the figure 3. The air movement is absent in case of heat conduction and the steady temperature distribution has linear dependence. This corresponds to the model of air heat conduction with constant physical features.

The influence of natural convection on the temperature distribution can be valued according the results of numerical modeling which are represented on the picture $3(G r>0)$.

Arithmetic mean of temperature deviation in the similar space points from the linear profile grows from 0.048 at $G r=5000$ to 0.1899 at $G r=200000$ in the absolute values. Extremal deviation meanings reach 0.0903 at $G r=5000$ and 0.4201 at $G r=200000$. The further growth of Grashof number leads to the greater deviation value of temperature from linear distribution. 


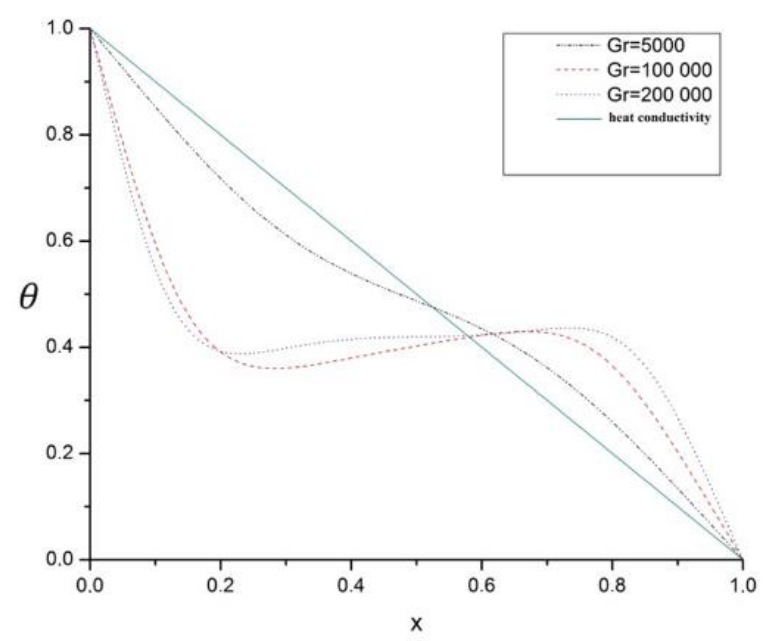

Fig. 3. Temperature distributions in the midsection $(y=1)$ at various Grashof numbers.

The received results are shown that significant temperature distributions' deviations from linear profile appear when considering the system "the heat source - environment - the object of heating". Therefore, the natural convection component of heat transfer when designing of microclimate's thermal conditions in rooms with heat sources should be taken into account.

\section{Conclusion}

Mathematical modeling of temperature fields in the system "the heat source - environment the object of heating" was carried out.

The problem of heat transfer between the object and the source in conditions of thermogravitational convection at small Grashof numbers was solved.

The natural convection influence to temperature fields in the system "the heat source environment - the object of heating" comparing with heat conduction model was evaluated.

\section{References}

1. P. J. Roache, Fundamentals of Computational Fluid Dynamics (Hermosa Publishers, Albuquerque, 2003)

2. A. A. Samarskii, The Theory of Difference Schemes (Marcel Dekker, New York, 2001)

3. H. K. Versteeg, W. Malalasekera, An introduction to computational fluid dynamics (Longman group, New York, 2007)

4. S. V. Patankar, Numerical Heat Transfer and Fluid Flow (CRC Press, Florida, 1980)

5. A. I. Vinogradova, V. G. Zubkov, Mathematical simulation E 6, 3 (2002) 